\author{
E. Athanassoula \\ Observatoire de Besançon and European Southern Observatory \\ M.F. Duval \\ Observatoire de Marseille
}

NGC 5383 has often been used as a prototype of SB(s)b type galaxies, particulary in studies of gas flow. We have therefore set out to complement the existing data with surface photometry, velocity measurements in the bar and a dynamical model.

The surface photometry of this galaxy shows the existence of four components: a bulge, a disk, a bar and a lens (Fig. 1). Their geometrical parameters are given below:

\begin{tabular}{lcc}
\hline & $\begin{array}{c}\text { position of the } \\
\text { major axis }\end{array}$ & $<\mathrm{b} / \mathrm{a}\rangle=\mathrm{q}$ \\
\hline bulge & $90^{\circ} \pm 5^{\circ} \mathrm{E}$ & $0.71 \pm 0.04$ \\
bar & $135^{\circ} \pm 2^{\circ} \mathrm{E}$ & $0.38 \pm 0.02$ \\
lens & $107^{\circ} \pm 2^{\circ} \mathrm{E}$ & $0.88 \pm 0.02$ \\
disk & $85^{\circ} \pm 3^{\circ} \mathrm{E}$ & $0.66 \pm 0.03$
\end{tabular}

Their effective luminosity distributions are described for the bulge and the disk by the classical formulas:

$\begin{array}{ll}\text { bulge } \log I_{b}=0.08-3.33\left[\left(\mathrm{r}^{*} / 0.16\right)^{1 / 4}-1\right] \mathrm{I}_{\mathrm{o}_{\mathrm{b}}}=3.210^{5} \mathrm{~L}_{\odot} \mathrm{pc}^{-2} \\ \text { disk } \log \mathrm{I}_{\mathrm{d}}=-0.9-0.456 \mathrm{r} * & \mathrm{I}_{\mathrm{o}_{\mathrm{d}}}=15.5 \mathrm{~L}_{\odot} \mathrm{pc}^{-2}\end{array}$

We obtain: $\mathrm{L}_{\mathrm{b}} / \mathrm{L}_{\mathrm{T}}=0.32$ and $\mathrm{L}_{\mathrm{d}} / \mathrm{L}_{\mathrm{T}}=0.33$

From the luminosity profiles of the bar and the lens, we deduce the best representative simple laws:

bar $I_{B}=0.42\left(1-\left(r^{*} / 0.60\right)^{2}\right)^{1 / 2}$

lens $I_{\ell}=0.18\left(1-\left(r^{*} / 1.35\right)^{2}\right) \quad$ for $r^{*}>0 ! 6$ and

$$
\mathrm{I}_{\mathrm{O}_{\mathrm{B}}}=51.9 \mathrm{~L}_{0} \mathrm{pc}^{-2}
$$$$
I_{\ell}=0 . \quad \text { for } r^{*}<0 ! 6
$$

( $r^{*}$ in arcmin)

So that: $\mathrm{L}_{\mathrm{B}} / \mathrm{L}_{\mathrm{T}}=\mathrm{L}_{\ell} / \mathrm{L}_{\mathrm{T}}=0.15$ 


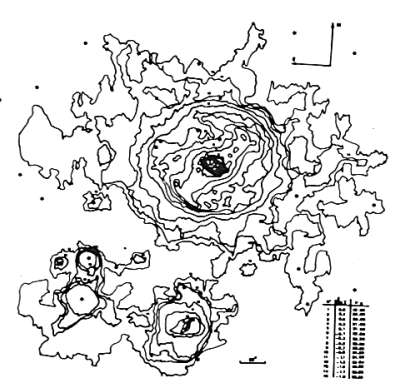

Figure 1. Isophotal map in blue light

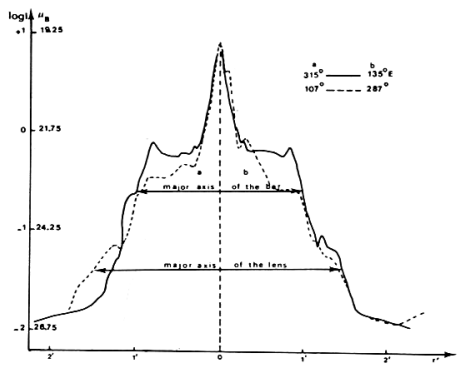

Figure 2. Luminosity profiles

Several spectra have been taken with slit positions complementing those of Duval (1977) and Peterson et al. (1978), thus providing good coverage of the bar region despite the fact that the bar does not contain much ionized gas.

Using the photometric properties of the four components, a set of models has been built and the velocity fields calculated with the help of the beam scheme, and compared to the observations. From the best fitting model we deduce the $M / L$ ratios of each component after correction for galactic and internal absorption :

$\begin{array}{llll}\begin{array}{lll}\text { bulge } \\ 1.4 \mathrm{~h}^{-1}\end{array} & \begin{array}{l}\text { bar } \\ 6.5 \mathrm{~h}^{-1}\end{array} & \begin{array}{l}\text { lens } \\ 9.3 \mathrm{~h}^{-1}\end{array} & \begin{array}{l}\text { disk }+ \text { halo } \\ 27 \mathrm{~h}^{-1}\end{array}\end{array} \quad$ in $\mathrm{M}_{\odot} / \mathrm{L}_{\odot}$

(distance : $23.5 \mathrm{~h} \mathrm{Mpc}$ and $\mathrm{h}=100 / \mathrm{H} \mathrm{km} \mathrm{s}^{-1} \mathrm{Mpc}^{-1}$ )

We note the similarity of the M/L ratios of the bar and the lens. Various "rotation curves" (Fig. 3) issued from the adopted model have been compared to the axisymmetric curve derived from HI data (Sancisi et a1., 1979).

\section{Figure 3.}

\section{REFERENCES}

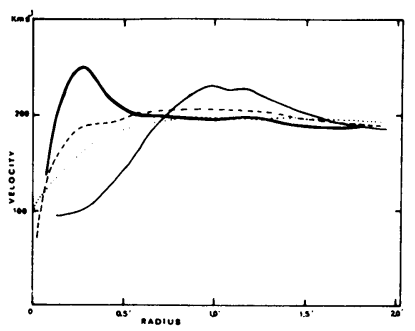

- cut along the bar

- cut across the bar

--- rotation velocities meaned in annuli

... Axisymmetric rotation curve from HI data

Duva1, M.F. : 1977, Astrophys. Space Sci. 48, 103.

Peterson, C.J., Rubin, V.C., Ford, W.K., Thonnard, N.: 1978, Astrophys. J. 219,31 .

Sancisi, R., Allen, R.J., Sullivan, W.T.: 1979, Astron. Astrophys. 78, 217. 